\title{
Identifying adverse drug events in patients at a pediatric ward in a Brazilian hospital: application and performance of the triggers
}

Simone Cristina Rodrigues de Carvalho Caetano 1

(iD https://orcid.org/0000-0003-2071-672X

Luiz Fernando Emidio da Silva 2

(iD https://orcid.org/0000-0003-0280-9105

Lusiele Guaraldo 3

iD https://orcid.org/0000-0003-0047-6538

Fabíola Giordani 4

iD https://orcid.org/0000-0003-2919-856X

\footnotetext{
1,2 Instituto Nacional de Saúde da Mulher, Criança e do Adolescente Fernandes Figueira. Fundação Oswaldo Cruz. Av. Rui Barbosa, 716. Flamengo. Rio de Janeiro, RJ, Brasil. CEP: 20.021-140. E-mail: simone.farma@gmail.com

3 Instituto Nacional de Infectologia Evandro Chagas. Fundação Oswaldo Cruz. Rio de Janeiro, RJ, Brasil

4 Universidade Federal Fluminense. Niterói, RJ, Brasil.
}

\begin{abstract}
Objectives: to evaluate the performance of a trigger tool in identifying adverse drug events (ADE) in hospitalized children.

Methods: a retrospective cohort study review on 133 medical records at a federal maternal and child reference hospital in Rio de Janeiro in 2016. A list of 14 triggers was developed to detect $A D E$ in the pediatric population. Three steps were performed: (1) search for triggers; (2) selection of suspected cases of $A D E$ and (3) final determination of $A D E$ by experts' consensus.

Results: 360 triggers were identified in 100 hospitalizations (75.2\%), with an average of 2.7 triggers/ hospitalization. The most frequent triggers were "abrupt medication stop" (79.7\%); "antiemetics use" (8.9\%) and "laxatives use" (7.2\%); while the "diphenhydramine use", "phytomenadione use" and "excessive sedation/lethargy/fall/hypotension" obtained the highest performance indicating ADE every time they occurred. Thirty-one ADE were identified in $12.8 \%$ of the hospitalizations; 11 (35.5\%) ADE were detected without the aid of the triggers thus, pruritus and diarrhea were the most frequent.

Conclusion: the trigger tool proved to be useful in identifying ADE in hospitalized children, especially if high performance and high frequency triggers are used in identifying the events. The inclusion of the triggers "diarrhea" and "pruritus", may favor the identification of $A D E$ in patients at pediatric wards.

Key wods Drug-related side effects and adverse reactions, Drug monitoring, Pediatric Hospitals, Hospitalization, Pharmacoepidemiology, Patient's safety
\end{abstract}

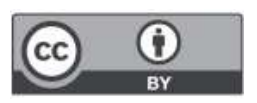




\section{Introduction}

Although healthcare is of fundamental importance, drug therapy may cause risks to patients, especially in the population groups with specific characteristics, such as children. 1,2

Most available drugs on the market are not tested in children during the pre-marketing period, since this age group is usually excluded from clinical trials to evaluate the efficacy and safety of new drugs. 1,3 In addition, few studies have been published on adverse events and other problems related to the use of drugs in pediatric patients compared to the studies in adults.4-6 The scarcity of studies on children contributes to the fact that pediatric prescriptions are supported by weak evidences. ${ }^{3}$ According to a study conducted with notification data made to the Agência Nacional de Vigilância Sanitária (ANVISA) (National Health Surveillance Agency) in the Brazilian pharmacovigilance system, between 2013 and 2016, 10.4\% of the suspected cases on adverse drug reactions reported were of children under 12 years of age, among which $60 \%$ corresponded to severe adverse reactions. 6

The World Health Organization (WHO) defines adverse drug events (ADE) as any injury or harm, causing by the use of one or more drugs for therapeutic purposes, therefore, can include adverse drug reactions (ADR) and medication errors. ${ }^{7}$

Among the strategies in identifying ADE, the voluntary reporting method is mostly used, due to its low cost and easy implementation, but its disadvantage is underreporting of cases. ${ }^{8}$ Thus, the incorporation of other methods is necessary to meet the challenges of the detection ADE.

A strategy that has been shown - in national9,10 and international studies 1,11 -efficiency in identifying ADE, is the use of triggers. This strategy was initially presented by the Institute for Healthcare Improvement (IHI), an American institution focused on improving quality and safety in healthcare, which proposed the use of triggers to identify events in hospitalized patients. 8,12 The method was developed in 2000 and comprises a retrospective review of a random sample of medical records seeking to identify ADE from a list of 19 triggers that act as "clues" on the events. 8,13 The triggers consist of medications, laboratorial test results, and patients' evolution data related to harmful medication use (ADE). 8,13 Takata et al. 14 adapted the method and it was developed by IHI to apply this tool in the pediatric inpatient settings.

Studies in the adult population have found that reviewing medical records to identify ADE with the aid of the triggers requires between 15 and 20 minutes, which is considered feasible. 13 The trigger tool allows to quantify adverse events (harms), using relatively small samples and tracking changes in the rate events over time.

This study aimed to evaluate the performance of the ADE trigger tool developed by IHI and adapted for the hospitalized pediatric population.

\section{Methods}

A retrospective cohort study was carried out by means on medical records review.

The study hospital is a public reference unit in maternal and child care, which develops assistance, teaching and research activities, located in the city of Rio de Janeiro. The hospital unit offers medium and high complexity services at a tertiary level of care, such as hospitalizations, surgeries, and consultations at specialized outpatient clinics. The profile of the population admitted to the pediatric ward is children and adolescents with chronic diseases and rare diseases (such as cystic fibrosis, mucopolysaccharidosis, osteogenesis imperfecta, genetic syndromes, and neurological diseases).

Medical records of patients under 13 years of age from the community, or from another hospital unit, or from another sector of the hospital itself, or admitted to the pediatric ward for more than 48 hours were analyzed. The follow-up period was 60 days of hospitalization, except in cases of discharge, death, or transferred to another service.

The sample size was calculated considering the estimated proportion of pediatric patients with the possibility of $20 \%$ of ADE, confidence level of $95 \%$ and precision of $5 \%$, obtaining a number of 133 admissions, corresponding to 110 patients. Readmissions were considered independently of the events. The simple random sample was selected from the admissions to the pediatric ward during the period and met the inclusion criteria.

The list of pediatric triggers proposed by Takata et al. ${ }^{14}$ was adapted according to the characteristics of the institution. For this, drug formulary approved by hospital and the available laboratorial tests were considered. The applicability of the triggers in the unit and the parameters for laboratorial test results were evaluated by the multidisciplinary team (physician, nurse and pharmacist) at the hospital, which decided to remove the "called code" trigger (medical records in ADE related to emergencies) due to the lack of this practice in the hospital, and for the substitution of the trigger "serum glucose $>150$ $\mathrm{mg} / \mathrm{dL}$ " for "hypoglycemia" as it was considered by 
the team, as a factor with a greater impact on the study population. No triggers were added to the initial list proposed for the data collection.

Thus, 14 triggers were selected, divided into four categories - medication use (7), clinical signs and symptoms (2), laboratorial test results (4), and actions taken by the clinical team (1). The selected triggers and rationale proposed by $\mathrm{IHI}^{14,15}$ are described in Table 1.

In order to standardize the data collection and solve eventual doubts about the application of the method, the professionals were previously trained and explanatory scripts were elaborated to adequately fill out the collection instruments.

The identification of ADE was carried out in three stages: (1) search for triggers; (2) selection of suspected cases of ADE and (3) a final determination of ADE through a consensus meeting among specialists.

The institution had no electronic medical records, and they were all handwritten. The review on the medical records obeyed the following order as suggested by IHI (2008): review of drug prescriptions, laboratorial test results, and clinical evaluation data performed by a multiprofessional team. 12

Initially, all medications prescribed/used by the patients were registered in a proper form with the start and end of use dates, for a better correlation of the triggers with use of the drugs. Afterwards, the triggers for $\mathrm{ADE}$ were identified.

In the following step, the medical records were analyzed to determine, for each identified trigger, the occurrence of an event associated with the use of a drug, ruling out possible alternative causes of the drug(s). Thus, the possible ADE algorithm was classified by Naranjo et al., 16 considering the temporal relationship between the event and the medication, the patient's clinical condition and pharmacological plausibility, with the help of specialized literature (Micromedex ${ }^{\circledR}$ Healthcare Series 17 and Meyler's side effects of drugs ${ }^{18}$ ). Only the cases classified by the algorithm as definite probable or possible were considered suspected cases. 16

The suspected cases of adverse events were then to an expert consensus to evaluate and define by consensus, of the occurrence of ADE. The group of experts was composed with a main researcher, a pediatrician, and an experienced pharmacist in pharmacovigilance and healthcare in the study population.

The severity of ADE was classified using the National Coordinating Council for Medication Error Reporting and Prevention (NCC MERP) categories, which included: harm (E: temporary harm to patient and need for intervention; F: temporary harm to patient requiring or prolonging hospitalization; G: permanent harm to patient; $\mathrm{H}$ : need for lifesustaining intervention; I: death). ${ }^{19}$

Data were processed using Epi Data 3.0 and analyzed using the $\mathrm{R}$ software version 3.3.2.

Descriptive analysis was performed considering ADE, the participants, and the triggers. The participants' characteristics were presented with a statistic summary where quantitative variables were represented by median and interquartile range and qualitative variables by absolute and relative frequencies. The indicators of ADE frequency were the incidence of patients with adverse drug events during hospitalization and the rate of ADE per 100 hospitalizations. The relative and absolute frequencies of events were presented according to the categories of severity.

The performance of the triggers was analyzed by three components: (1) frequency of each trigger identified per 100 hospitalizations; (2) frequency of events identified per 100 hospitalizations; (3) relative percentage of performance of each trigger $((2) /(1))$. The calculated performance was categorized into three groups - high $(100 \%)$, intermediate (between 30 and $70 \%$ ), and low $(<30 \%) \cdot 9,10$

This present study was approved by the Research Ethics Committees at Instituto Nacional de Saúde da Mulher, da Criança e do Adolescente Fernandes Figueira and at Hospital Universitário Antônio Pedro, registry numbers (CAAE) 68461417.7.0000.5243 and 68461417.7.3001.5269.

\section{Results}

A total of 133 hospitalizations of 110 patients were analyzed, among which $100(75.2 \%)$ had at least one trigger obtaining an average of 2.7 triggers per hospitalization (Figure 1).

Patients had median ages of 1.7 years (IIQ: $<1.0$ 3.8 years) and the median length of hospitalization was 6 days (IIQ: 3.0-14.0) and the median number of medications used during hospitalization was 4.1 (IIQ: 2.2-6.1). Most patients were male (70; 52.6\%) and mixed color skin $(68 ; 51.1 \%)$. Three main diagnoses were highlighted among the analyzed hospitalizations: cystic fibrosis $(14 ; 10.5 \%)$, other bacterial pneumonias $(14 ; 10.5 \%)$, and pneumonias due to other microorganism infection (12;9.0\%).

A total of 31 ADE was identified in 17 hospitalizations, corresponding to a rate of 23.3 ADE per 100 hospitalizations. ADE were identified in $12.8 \%$ of the hospitalizations. Of the 31 events, 29 (93.6\%) were classified in category E, i.e. caused by temporary harm to the patient and the need for interven- 
Table 1

List of triggers on pediatric adverse drug event (ADE) used in the study and rationale for investigating ADE.

Triggers

Description and rationale

Observations

Medications

Diphenhydramine use

Phytomenadione use

Flumazenil use

Antiemetic use

Naloxone use

Calcium polystyrenesulfonate use

Laxatives use

Clinical signs and symptoms

Excess sedation/ lethargy/ fall/ hypotension

Skin rash

Laboratorial Test Results

Partial Thromboplastin Time (PTT)

Increased serum creatinine

Hypoglycemia

Hyperkalemia

Clinical conduct

Abrupt medication stop
Antihistamine. May have been used to treat allergic reaction caused by the medication.

Antihemorrhagic. May have been used to treat signs of bleeding caused by the medication.

Antidote for benzodiazepine. Check medical records for hypotension or prolonged sedation after benzodiazepine use.

Requires careful analysis to rule out alternative causes that prompted the use of antiemetics and to determine if there was actually an ADE.

Opioid antidote. Check medical records for signs of opioid toxicity, such as respiratory depression, nausea and vomiting, constipation, and urinary retention.

Ion exchange resin used in the treatment of hyperkalemia. Correlate drug use with elevated serum potassium levels.

Search for evidence of medication-induced constipation in prescriptions and in patient's clinical evaluation records.

Search for the relation between these events and the administration of sedative, analgesic, or muscle relaxer medications.

Check medical record for a correlation between the event and the administration of a medication.

Verify the correlation between bleeding signs and the use of heparin.

Check for administration of nephrotoxic medication and exclude alternative causes of renal failure, such as pre-existing kidney disease or diabetes.

Correlate hypoglycemia with medication use.

Check for correlation of hyperkalemia with medication use.

Interruption of a medication may indicate the occurrence of $A D E$. It is necessary to exclude alternative causes for the interruption of the medication.
The triggers that refer to the use of a medication are identified in the prescriptions. The reasons for the use of the medication should be identified with a view to ruling out alternative causes and confirming the event

Search the clinical evaluation records performed by the assistance team

PTT > 100 seconds

Alteration $>0.4 \mathrm{mg} / \mathrm{dL}$

Blood glucose $<40 \mathrm{mg} / \mathrm{dL}$

Values > $5.5 \mathrm{mg} / \mathrm{dL}$

The identification of this trigger considered the use of the word "suspended" in the prescription. 
tion, one $(3.2 \%)$ was classified as $\mathrm{F}$ caused by temporary harm to the patient and need for initial or prolonged hospitalization, and one (3.2\%) was classified as G, permanent harm was caused to the patient.

Among the 360 triggers identified, 287 (79.7\%) were "abrupt medication stop", 32 (8.9\%) "antiemetics use", 26 (7.2\%) "laxatives use", 4 $(1.1 \%)$ "phytomenadione use", $3(0.8 \%)$ "rash" and 3 (0.8\%) "hyperkalemia", 2 (0.6\%) "excessive sedation/lethargy/fall/hypotension" and the trigger detected 1 time $(0.3 \%)$ "diphenhydramine use", "naloxone use" and "hypoglycemia". The triggers "flumazenil use", "calcium polystyrenesulfonate use", "partial thromboplastin time", and "increased serum creatinine" were not identified in this study.

Of the identified triggers, "abrupt medication stop " was mostly related to ADE ( $\mathrm{n}=7)$; followed by the "phytomenadione use" $(n=4)$, "laxative use" $(n=3), \quad$ "excessive sedation/lethargy/fall/ hypotension"( $\mathrm{n}=2)$, "rash" $(\mathrm{n}=2)$ and "diphenhydramine use" ( $\mathrm{n}=1)$. "naloxone use", "hypoglycemia" and "hyperkalemia" were not associated with ADE.

Regarding relative performance of the triggers, "diphenhydramine use", "phytomenadione use", and "excessive sedation/lethargic/fall/hypotension" were considered high performance $(100 \%)$. The triggers "rash" achieved intermediate performance (66.7\%) and the "laxatives use" (11.5\%), "antiemetics use" (3.1\%) and "abrupt medication stop" (2.4\%) showed low performance (Table 2).

Among the $31 \mathrm{ADE}$ identified, 11 (35.5\%) were not related to the use of the triggers, among these,

\section{Figure 1}

Representative flowchart of the steps of adverse drug events (ADE) identified by the trigger tool in the patients' medical records at a federal reference hospital in maternal and child care in Rio de Janeiro, 2016.

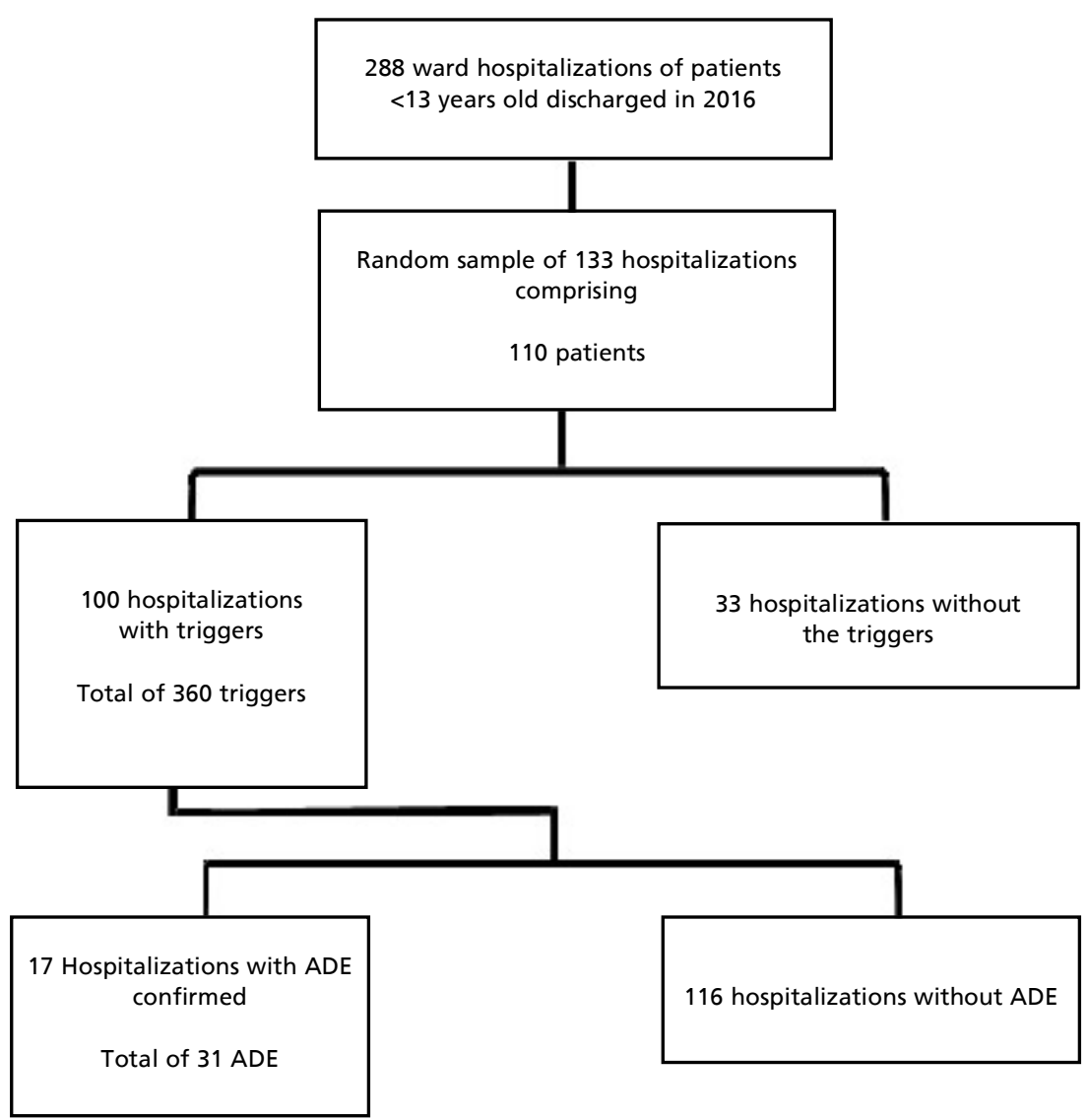


three were identified in the medical record registered as "itching during/after infusion" of the medication and two were registered "diarrhea associated with antibiotic use". The others were registered "significant sialorrhea after oral antibiotic use", "medium nasal bleeding", "insomnia", "phlebitis", "worsening myoclonus" and hepatitis medications.

\section{Discussion}

The trigger tool used proved to be applicable and useful in identifying adverse drug events in hospitalized children in the pediatric ward of a federal teaching hospital, identifying ADE in $12.8 \%$ of the analyzed hospitalizations. The most frequent trigger responsible for detecting the highest number of ADE was "abrupt medication stop", while the "diphenhydramine use", "phytomenadione use" and "excessive sedation/lethargy/fall/hypotension", indicated ADE whenever present.

In this study, the most frequent triggers found were "abrupt medication stop" "antiemetics use," and "laxatives use." Similar data were found in another study that used the same tool and a pediatric population. 20 The detection of the "abrupt medication stop" trigger requires a careful analysis for a proper interpretation as to the occurrence or not of ADE. There are reasons that lead to the interruption of the medication that are unrelated to the occurrence of an adverse event, such as treatment termination, dose alteration, and improvement in the patient's clinical condition. The proper application of this trigger requires the definition of criteria based on the profile of the healthcare facility and the techniques used for data collection. Similarly, the trigger the "antiemetics use" and "laxatives use" refers to drugs that appear quite frequently in prescriptions but may be related to other common indications for patients with the profile of the study institution.

The trigger "flumazenil use", "calcium polystyrenesulfonate use", "partial thromboplastin time", and "increased serum creatinine" were not identified in any hospitalization. It is possible that they could demonstrate utility in larger samples or in pediatric populations with a more extended profile (emergency, surgical patients, intensive care units, etc.).

Regarding to the events identified, the triggers on "abrupt medication stop", "phytomenadione use", and "laxatives use" identified the highest number of ADE. This result was expected since these triggers occurred at a higher frequency. The triggers "abrupt medication stop" has shown similar performance in studies with adults and newborns. 10,21,22

Comparing the ability to detect ADE of each trigger, three groups were observed. The ones that showed the best performance were "excessive seda-

\section{Table 2}

The performance on adverse drug event (ADE) of triggers found in medical records of patients under 13 years of age, discharged between January and December 2016, in a federal reference hospital in maternal and child care in Rio de Janeiro.

\begin{tabular}{lccc}
\hline Trigger/Performance & $\begin{array}{c}\text { Triggers per 100 } \\
\text { hospitalizations* } \\
(1)\end{array}$ & $\begin{array}{c}\text { ADE per 100 } \\
\text { hospitalizations* } \\
\text { (2) }\end{array}$ & $\begin{array}{c}\text { Relative performance } \\
\text { of the riggers } \\
(3)=(2))_{(1)} \times 100\end{array}$ \\
\hline High performance & & & \\
$\quad$ Diphenhydramine use & 0.8 & 0.8 & 100.0 \\
Phytomenadione use & 3.0 & 3.0 & 100.0 \\
Excess of sedation/ lethargy/ fall/ hypotension & 1.5 & 1.5 & 100.0 \\
& & & \\
Intermediate performance & & & 66.7 \\
Skin rash & 2.3 & 1.5 & \\
& & & 11.5 \\
Low performance & 19.5 & 2.3 & 3.1 \\
Laxatives use & 24.1 & 0.8 & 2.4 \\
Antiemetics use & 215.8 & 5.3 & 0.0 \\
Abrupt medication stop & 0.8 & 0.0 & 0.0 \\
Naloxone use & 0.8 & 0.0 & 0.0 \\
Hypoglycemia & 2.3 & 0.0 & \\
Hyperkalemia & & & \\
& &
\end{tabular}

* Total hospitalizations evaluated $=133$ 
tion/lethargy/fall/hypotension", "diphenhydramine use", and "phytomenadione use". The first one highlighted as a high-performance trigger in two other studies conducted with the pediatric population. 10,14 The triggers "diphenhydramine use" and "phytomenadione use", besides their high-performance, were easy to identify in the patient's chart, especially when the hospital has an electronic prescription system. Also, the "diphenhydramine use" identified one of the ADE that was classified with a high level of harm (F). Thus, it is suggested that the implementation of an ADE monitoring program should be initiated using these triggers.

In the intermediate performance group found the "rash" trigger with an approximately of $67 \%$. Identifying ADE from the trigger requires a detailed investigation to establish the causal relationship between the administration of the drug and the occurrence of the rash and depends on the quality of the registrations. In comparison with other studies, the trigger "rash" showed equivalent performance as identified here23; it appeared among the six highest performances and showed low performance in a Brazilian study that analyzed ADE performance in newborns. 10

The last group consisted by the triggers had a performance below 30\%: the "laxatives use", "antiemetics use" and "abrupt medication stop". Despite the low performance, the triggers "laxatives use" and "abrupt medication stop" indicated half of the ADE identified, which makes them useful for monitoring events. The triggers "laxatives use" and "antiemetics use" obtained low performance in another study with a pediatric population. 14 Antiemetics are used for nausea and vomiting, nonspecific symptoms and very common in various clinical conditions, which makes it difficult to determine the causal relationship with the use of the medications.

Among the ADE identified, 11 were captured through the multidisciplinary team's notes in the medical record without, however, being identified by the triggers. Of these, pruritus was identified in three medical records and diarrhea was identified in two. In the three cases of pruritus, hydroxyzine, an antihistamine was used for allergies. This suggests that replacing the "diphenhydramine use" with the "antihistamine use" as a trigger, it would make it more comprehensive and would increase the ability to detect ADE. A Brazilian study used "antihistamines use" and observed a high positive predictive value for this trigger. 24 In addition, the inclusion of the triggers "diarrhea" and "pruritus" could increase the ability to identify ADE in the study population.
In the severity assessment according to the NCC MERP index, 29 of 31 ADE identified (93.6\%) were classified in the milder damage category (E). This result is similar to those found in Takata et al.14,25 (2008) (97.2\% and 93.7\%), and Burch 20 (2011) (94.1\%) studies, who also used the trigger to identify the events in children.

This present study has some limitations. The retrospective review of the medical records use the existing medical records carried out by several professionals, not always in a standardized manner, presenting sometimes missing or poorly detailed data.

Another limitation was due to the fact that the study was carried out in only one reference hospital for child health and may not be generalizable or applied to other hospitals with different profiles.

Among the strengths of this study, there were the selection of medical records by random sample, standardized collection of drugs prescribed/ used, daily, during hospitalization, and the participation of a multidisciplinary team in the stages of defining the triggers and confirming the ADE.

Thus, the results of this study suggest that the trigger tool has proven to be useful for monitoring adverse drug events in patients at the pediatric ward, especially if high performance and high frequency triggers are used to identify the events. In addition, the inclusion of the triggers "diarrhea" and "pruritus" may favor the identification of ADE in patients at the pediatric ward.

The retrospective analysis of the medical records in identifying ADE is a time-consuming process, especially when the system is not computerized. The use of the trigger tool, therefore, helps to direct the analysis by focusing on the search of specific points in the medical records, allowing to achieve better results in detecting rates of the events. ${ }^{11}$

\section{Acknowledgments}

We would like to thank the Post Graduation Program in Medical Sciences at the Faculdade de Medicina da Universidade Federal Fluminense, where the first author developed the study to obtain her Master's degree, and to the Coordenação de Aperfeiçoamento de Pessoal de Nivel Superior (CAPES) for the Master's scholarship granted to the first author.

\section{Authors' contribution}

Caetano SCRC participated in the conception, data collection, data analysis and interpretation, article writing and approval of the final version. Silva LFE 
participated in the data collection. Guaraldo L participated in the conception, article writing, and approval of the final version. Giordani F participated

\section{References}

1. Kaushal R, Jaggi T, Walsh K, Fortescue EB, Bates DW. Pediatric medication errors: what do we know? What gaps remain? Ambul Pediatr. 2004; 4 (1): 73-81.

2. Liu Y, Yan J, Xie Y, Bian Y. Establishment of a pediatric trigger tool based on Global Trigger Tool to identify adverse drug events of children: experience in a Chinese hospital. BMC Pediatr. 2020; 29; 20 (1): 454.

3. Mir AN, Geer MI. Off-label use of medicines in children. Int J Pharm Sci Res. 2016; 7 (5): 1820-8.

4. Kaushal R, Bates DW, Landrigan C, McKenna KJ, Clapp MD, Federico F, Goldmann DA. Medication errors and adverse drug events in pediatric inpatients. JAMA. 2001; 285 (16): 2114-20.

5. Buck ML. Adverse Drug Events in Children: Recent Cases from the Medical Literature. Pediatr Pharmacother. 2010 $16(9)$

6. Lima EC, Matos GC, Vieira JML, Gonçalves ICCR, Cabral LM, Turner MA. Suspeitas de reações adversas a medicamento relatadas em crianças brasileiras: estudo transversal. J Pediatr (Rio J.). 2019; 95 (6): 682-8.

7. WHO (World Health Organization). The Conceptual Framework for the International Classification for Patien Safety (ICPS) [Internet]. Geneva; 2009 [cited 2020 May 15]. Available from: https://apps.who.int/iris/bitstream/ handle/10665/70882/WHO_IER_PSP_2010.2_eng.pdf?seq uence $=1$

8. Griffin FA, Resar RK. IHI Global Trigger Tool for Measuring Adverse Events (Second Edition). IHI Innovation Series white paper. Cambridge, MA: Institute for Healthcare Improvement; 2009. [cited 2021 Aug 10]. Available from: www.IHI.org

9. Giordani F, Rozenfeld S, Martins M. Adverse drug events identified by triggers at a teaching hospital in Brazil. BMC Pharmacol Toxicol. 2014; 15: 71.

10. Fabretti S de C, Brassica SC, Cianciarullo MA, RomanoLieber NS. Rastreadores para a busca ativa de eventos adversos a medicamentos em recém-nascidos. Cad Saúde Pública. 2018; 34 (9): e00069817.

11. Kirkendall ES, Kloppenborg E, Papp J, White D, Frese C, Hacker D, Schoettker PJ, Muething S, Kotagal U. Measuring adverse events and levels of harm in pediatric inpatients with the Global Trigger Tool. Pediatrics. 2012; 130 (5): e1206-1214

12. IHI (Institute for Healthcare Improvement). Introduction to Trigger Tools for Identifying Adverse Events. 2008. [cited 2021 Aug 10]. Available from: www.IHI.org

Received on October 20, 2020

Final version presented on August 17, 2021

Approved on September 29, 2021 in the conception, data analysis and interpretation, article writing and approval of the final version.

13. Rozich JD, Haraden CR, Resar RK. Adverse drugevent trigger tool: a practical methodology for measuringmedicationrelatedharm. Qual Saf Health Care. 2003; 12(3): 194200.

14. Takata GS, Mason W, Taketomo C, Logsdon T, Sharek PJ. Development, testing, and findings of a pediatric-focused trigger tool to identify medication-related harm in US children's hospitals. Pediatrics. 2008; 121 (4): e927-35.

15. IHI (Institute for Healthcare Improvement). Pediatric Trigger Toolkit: Measuring Adverse Drug Events in the Children's Hospital [Internet]. 2007 [cited 2020 May 15]. http://www.ihi.org/resources/Pages/Tools/PediatricADETri ggerToolkit.aspx

16. Naranjo CA, Busto U, Sellers EM Sandor P, Ruiz I, Roberts EA, et al. A method for estimating the probability of adverse drug reactions. Clin PharmacolTher. 1981; 30 (2): 239-45.

17. Micromedex Healthcare Series [Internet]. Greenwood Village: Thomson Healthcare; 2006. Available from: https://rdl.lib.uconn.edu/databases/919

18. Desai C. Meyler's side effects of drugs: The international encyclopedia of adverse drug reactions and interactions. Indian J Pharmacol. 2016; 48 (2): 224.

19. NCC MERP (National Coordinating Council for Medication Error Reporting and Prevention). Index for categorizing medication errors. [cited 2021 Aug 10]. Available from: https://www.nccmerp.org/types-medication-errors

20. Burch KJ. Using a Trigger Tool to Assess Adverse Drug Events in a Children's Rehabilitation Hospital. J Pediatr Pharmacol Ther. 2011; 16 (3): 204-9.

21. Sam AT, Lian Jessica LL, Parasuraman S. A retrospective study on the incidences of adverse drug events and analysis of the contributing trigger factors. J Basic Clin Pharm. 2015; 6 (2): 64-8.

22. Giordani F, Rozenfeld S, Oliveira DFM, Versa GLGS, Terencio JS, Caldeira LF, Andrade LCG. Vigilância de eventos adversos a medicamentos em hospitais: aplicação e desempenho de rastreadores. Rev Bras Epidemiol. 2012; 15 (3): 455-67.

23. Hu Q, Qin Z, Zhan M, Chen Z, Wu B, Xu T. Validating the Chinese geriatric trigger tool and analyzing adverse drug event associated risk factors in elderly Chinese patients: A retrospective review. PLoS One. 2020; 15 (4): e0232095.

24. Silva LT, Modesto ACF, Martins RR, Bessa GG, Lopes FM. The Brazilian Portuguese version of the Pediatric Trigger Toolkit is applicable to measure the occurrence of adverse drug events in Brazilian pediatric inpatients. J Pediatr (Rio J). 2019; 95 (1): 61-8.

25. Takata GS, Taketomo CK, Waite S. Characteristics of medication errors and adverse drug events in hospitals participating in the California Pediatric Patient Safety Initiative. Am J Health Syst Pharm. 2008; 65 (21): 203644. 